AS-ITP-2003-002

\title{
A QCD Analysis of Quark Recombination for Leading Particle Effect
}

\author{
Chao-Hsi Chang, Jian-Ping Ma \\ Institute of Theoretical Physics, Academia Sinica, Beijing 100080, China \\ Zong-Guo Si \\ Department of Physics, Shandong University, Jinan Shandong 250100, China
}

\begin{abstract}
The quark recombination mechanism is proposed to explain the asymmetry between production rates of $D^{+}$and of $D^{-}$in their inclusive productions, and also asymmetries for other charmed hadrons. These asymmetries are observed in experiment and are called as leading particle effects. In this work we give a general analysis for contributions of quark recombination to these asymmetries. The contributions consist of a perturbative- and nonperturbative part. We perform two types of factorization by considering the produced hadron with large transverse momentum and by taking charm quark as a heavy quark, respectively. In the case of large transverse momentum the effect of quark recombination is the standard twist-4 effect. We find that the contributions are parameterized with four nonperturbative functions, defined with four quark operators at twist-4, for initial hadrons without polarization. By taking charm quark as a heavy quark the factorization can be performed with the heavy quark effective theory(HQET). The effect of quark recombination is in general parameterized by eight nonperturbative parameters which are defined as integrals with matrix elements of four quark operators defined in HQET. For unpolarized hadrons in the initial state, the parameters can effectively be reduced to four. Perturbative parts in the two types of factorization are calculated at tree-level.
\end{abstract}

PACS numbers: 12.38.Bx, 12.39.Hg, $13.85 . \mathrm{Ni}$ 


\section{Introduction}

Because nonperturbative physics of QCD is still unclear, a prediction of inclusive hadroproduction of a hadron can not be made in general. However, if the produced hadron has a large transverse momentum $k_{t}$, one can predict the differential cross section. According to the factorization theorem in $\mathrm{QCD}$ (see e.g., [1]), the prediction takes a form as a convolution with parton distributions for partons in the initial hadrons, fragmentation function of a produced parton decaying into the produced hadron, and perturbative functions for partonic processes. The prediction is based on the leading terms in the so called twist expansion. In the leading terms twist-2 operators are used to define nonperturbative parts, i.e., parton distributions and fragmentation functions. It can be shown that the next-to-leading orders are suppressed by certain power of $k_{t}$ relatively to the leading order. At the next-to-leading order, i.e., at twist-4 level, various partonic processes can happen. The nonperturbative effects are parameterized by matrix elements of twist-4 operators. At this order, a produced meson can be formed by recombination of two partons in a initial hadron. This is the so called parton recombination model [2], which is proposed to predict the inclusive production of a meson with low $k_{t}$. The recombination can also happen with one of the two parton involved in a partonic process. This has been studied in [3].

If we take the produced hadron containing one heavy quark in a hadron collision, for example, $D^{+}$, and neglect possible $c$-quark content in the initial hadrons, then at leading order of perturbative QCD the $D^{+}$production can be understood as that a $c \bar{c}$ pair is produced via partonic processes like $g g \rightarrow c \bar{c}$ or $q \bar{q} \rightarrow c \bar{c}$ and then the $c$-quark fragments into the $D^{+}$meson. It is clear that the production rate for $D^{+}$and for $D^{-}$is the same at this order. Experimentally significant asymmetries between these production rates for a class of c-flavored hadrons have been observed and have been called as leading particle effects. These effects are observed in fixed-target hadroproduction experiments for the production of c-flavored hadrons $[4, ?, ?, ?, ?]$ and also in photoproduction experiments [9-11]. Significant asymmetries are observed for $D^{+}-D^{-}, D^{0}-\bar{D}^{0}$ and $D^{*+}-D^{*-}$, while for $D_{s}^{+}$and $\Lambda_{c}^{+}$the asymmetries with large errors are consistent with zero. These asymmetries are large when $k_{t}$ is small, for $D^{+}$and $D^{-}$it can be $\sim 0.7$ in the forward direction in hadroproduction. Theoretically, various explanations exist [12,13], e.g., these asymmetries can be generated by intrinsic charm in the initial hadrons, but the predicted asymmetries are smaller than those in experiment. It should be noted that these asymmetries can also be generated at next-to-leading order of perturbative QCD [14-17], the predicted asymmetries are too small to explain the observed.

Recently, it has been suggested that these leading particle effects can be explained with quark recombination [18-20]. In E791 experiment [4] a $\pi^{-}$beam is scattered on a nuclear target, a $D^{-}$meson can be produced through quark recombination of a produced $\bar{c}$-quark with the valence quark $d$ in $\pi^{-}$after hard parton scattering, while a $D^{+}$meson can be produced through quark recombination of a produced $c$-quark with the sea quark $\bar{d}$ in $\pi^{-}$. Because the distribution of $d-$ and $\bar{d}$ quark in $\pi^{-}$are significantly different, it results in that more $D^{-}$are produced than $D^{+}$ in the forward direction. Motivated by NRQCD factorization [21], the authors of [18-20] take a factorization approach for quark recombination, in which a $c$-quark combines with a $\bar{d}$ quark in the case for $D^{+}$meson and the combination $c \bar{d}$ is in ${ }^{1} S_{0}$ or ${ }^{3} S_{1}$ state, i.e., the pair $c \bar{d}$ is formed as a pseudoscalar state or vector state, the $c \bar{d}$ is then inclusively transformed into the $D^{+}$. If 
the $\bar{d}$ quark was a heavy quark, the above states would be dominant in the quark recombination. However, the $\bar{d}$ quark is a light quark, for it a NRQCD description is not correct. It is possible that the $c \bar{d}$ is produced in a scalar, pseudovector and tensor state and then transformed into $D^{+}$.

If one takes $c$-quark as a heavy quark, a factorization different than that mentioned at the beginning can be performed with the Heavy Quark Effective Theory(HQET) [22]. In the heavy quark limit $m_{c} \rightarrow \infty$, the c-flavored hadron produced in a collision will carry the most momentum of the produced $c$-quark which is transformed into the hadron after its production. This suggests a factorization for the production of the c-flavored hadron, and in this factorization the production rate can be written as a product of the production rate of $c$-quark and a matrix element defined in HQET, corrections to this are suppressed by certain power of $m_{c}^{-1}$. This factorization was used to predict heavy quark fragmentation functions [23] and spin alignment of heavy meson in its inclusive production [24]. With this factorization predictions for these two cases are in good agreement with experiment at $e^{+} e^{-}$colliders [23,24].

In this work we will take this factorization to analyze the possible source for generating leading particle effects. We find that in general the $c \bar{d}$ pair can be first formed into the states of scalar, pseudoscalar, vector and pseudovector, from these states a $D^{+}$can be generated by emitting unobserved states. Our final results consist of 8 nonperturbative parameters, which are defined with matrix elements of HQET. Corrections to our results are suppressed by the inverse of heavy quark mass. These parameters can be calculated with nonperturbative methods. For initial hadrons without polarizations, these 8 parameters can be effectively reduced to 4 because some perturbative coefficients of the parameters in their contributions to the production rate are same. If the initial hadrons are polarized, all perturbative coefficients can be different, the number of parameters can not be reduced. In the approach of [18-20] the contributions from quark recombination for unpolarized initial state also contain 4 nonperturbative parameters, but their interpretation is different than our 4 parameters obtained from the effective reduction of the 8 parameters. Our results hold not only for low $k_{t}$, but also for high $k_{t}$. If $k_{t}$ is enough large so that all quark masses can be neglected, the standard factorization mentioned at the beginning can be performed. In this work we also perform an analysis for this case. This is similar to the analysis of twist-4 effects in deeply inelastic scattering [25], however, our task here is simple, because we only need to analyze the contributions from twist-4 operators, which are defined with 4-quark operators. These twist-4 operators are corresponding to those 4-quark operators in deeply inelastic scattering. In our final results for large $k_{t}$, there are four nonperturbative functions defined with 4-quark operators separating along the light-cone. It should be noted that it is rather difficult to perform a factorization for the relevant processes, in which hadrons are in the initial- and final state. We will assume that the factorization holds and especially that the parton model can be used for initial hadrons. With this assumption we need only to consider inclusive production of a hadron in a parton collision and focus on the final hadron.

Our work is organized as the following: In Sect. 2. we give our notations and analyze the quark recombination in the case with large $k_{t}$ where the $k_{t}$ is so large that all quark masses can be neglected. The contribution to the differential cross-section is a convolution of perturbative functions for partonic process with nonperturbative functions defined with four quark operators. In Sect.3. we analyze the quark recombination with the mentioned factorization with HQET, in which nonperturbative parts are defined as matrix elements of HQET. There are eight nonperturbative 
parameters characterizing different states of a quark pair which is transformed into the produced hadron. We also show in detail that these 8 parameters in the case with unpolarized beams can be effectively reduced to 4 . Discussions of our results are given. Sect.4 is our summary.

\section{Quark Recombination at Large $k_{t}$ and Twist-4 Effect}

We will denote a heavy quark as $Q$ and a heavy meson containing one heavy quark $Q$ as $H_{Q}$. The heavy meson $H_{Q}$ has a valence quark $\bar{q}$. As discussed in the introduction we will consider the production of $H_{Q}$ in a parton collision and a initial parton is a valence parton of $H_{Q}$. Neglecting possible heavy quark content in initial hadrons, the relevant parton process for quark recombination is:

$$
g\left(p_{1}\right)+\bar{q}\left(p_{2}\right) \rightarrow H_{Q}(k)+X
$$

where momenta are given in the brackets. We can always divide the unobserved state into a nonperturbatively produced part $X_{N}$ and a perturbatively produced part $X_{P}$, i.e., $X=X_{N}+$ $X_{P}$. At leading order of $\alpha_{s}, X_{P}$ is just a antiquark $\bar{Q}$. The scattering amplitude for the quark recombination can be written:

$$
\mathcal{T}=\int \frac{d^{4} k_{1}}{(2 \pi)^{4}} A_{i j}\left(p_{1}, p_{2}, k_{1}, p_{3}\right) \int d^{4} x_{1} e^{-i k_{1} \cdot x_{1}}\left\langle H_{Q}+X_{N}\left|\bar{Q}_{i}\left(x_{1}\right) q_{j}(0)\right| 0\right\rangle,
$$

where the indices $i, j$ stand for color- and spin indices. $\bar{Q}\left(x_{1}\right)$ and $q(0)$ are the fields of $Q$ and $q$ respectively. $A_{i j}$ is the amplitude for $g\left(p_{1}\right)+\bar{q}\left(p_{2}\right) \rightarrow Q^{*}\left(k_{1}\right)+\bar{q}^{*}\left(k_{q}\right)+\bar{Q}\left(p_{3}\right)$, ${ }^{*}$ means that the states are not on-shell. If the states are on-shell, then the amplitude is $\bar{u}\left(k_{1}\right)_{i} v_{j}\left(k_{q}\right) A_{i j}$ with on-shell conditions for corresponding partons. The Feynman diagrams for the amplitude $A_{i j}$ are given in Fig.1. For the process we define the variables:

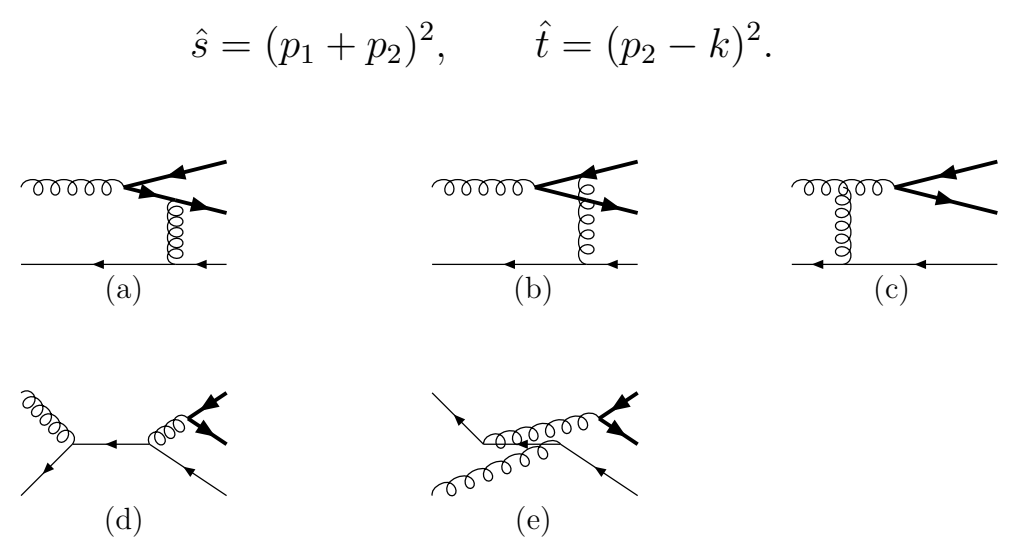

Fig.1. Feynman diagrams for the amplitude $A$.

The contribution from the process in Eq.(1) to the differential cross section is: 


$$
\begin{aligned}
d \sigma_{R}= & \frac{1}{2 \hat{s}} \sum_{X_{N}} \frac{d^{3} k}{(2 \pi)^{3} 2 k^{0}} \int \frac{d^{3} p_{3}}{(2 \pi)^{3} 2 p_{3}^{0}}(2 \pi)^{4} \delta^{4}\left(p_{1}+p_{2}-k-p_{3}-P_{X_{N}}\right) \\
& \cdot \int \frac{d^{4} k_{1}}{(2 \pi)^{4}} \frac{d^{4} k_{2}}{(2 \pi)^{4}} A_{i j}\left(p_{1}, p_{2}, k_{1}, p_{3}\right)\left(\gamma^{0} A^{\dagger}\left(p_{1}, p_{2}, k_{2}, p_{3}\right) \gamma^{0}\right)_{k l} \\
& \cdot \int d^{4} x_{1} d^{4} x_{2} e^{-i k_{1} \cdot x_{1}+i k_{2} \cdot x_{2}}\left\langle 0\left|\bar{q}_{k}(0) Q_{l}\left(x_{2}\right)\right| H_{Q}+X_{N}\right\rangle\left\langle H_{Q}+X_{N}\left|\bar{Q}_{i}\left(x_{1}\right) q_{j}(0)\right| 0\right\rangle,
\end{aligned}
$$

where we use the subscribe $R$ to denote the contribution from quark recombination, the average of spin and color of initial partons is implied. If $H_{Q}$ has nonzero spin, the summation over the spin is understood. Using translational covariance one can eliminate the sum over $X_{N}$. We define $a_{H_{Q}}^{\dagger}$ as the creation operator for $H_{Q}$ and we obtain:

$$
\begin{aligned}
d \sigma_{R}= & \frac{1}{2 \hat{s}} \frac{d^{3} k}{(2 \pi)^{3} 2 k^{0}} \int \frac{d^{3} p_{3}}{(2 \pi)^{3} 2 p_{3}^{0}} \cdot \int \frac{d^{4} k_{1}}{(2 \pi)^{4}} \frac{d^{4} k_{2}}{(2 \pi)^{4}} A_{i j}\left(p_{1}, p_{2}, k_{1}, p_{3}\right)\left(\gamma^{0} A^{\dagger}\left(p_{1}, p_{2}, k_{2}, p_{3}\right) \gamma^{0}\right)_{k l} \\
& \cdot \int d^{4} x_{1} d^{4} x_{2} d^{4} x_{3} e^{-i k_{1} \cdot x_{1}+i k_{2} \cdot x_{2}-i x_{3} \cdot k_{3}}\left\langle 0\left|\bar{q}_{k}(0) Q_{l}\left(x_{2}\right) a_{H_{Q}}^{\dagger}(k) a_{H_{Q}}(k) \bar{Q}_{i}\left(x_{1}\right) q_{j}\left(x_{3}\right)\right| 0\right\rangle,
\end{aligned}
$$

with $k_{3}=p_{1}+p_{2}-k_{1}-p_{3}$ as the momentum of $\bar{q}$ after the hard scattering in the amplitude $A_{i j}\left(p_{1}, p_{2}, k_{1}, p_{3}\right)$. This contribution can be conveniently represented by the diagram in Fig.2., where the lower part represents the perturbative part, i.e., $A_{i j}\left(p_{1}, p_{2}, k_{1}, p_{3}\right)\left(\gamma^{0} A^{\dagger}\left(p_{1}, p_{2}, k_{2}, p_{3}\right) \gamma^{0}\right)_{k l}$, the upper part with the black box represents the nonperturbative part, i.e., the Fourier transformed matrix element in the second line of the above equation.

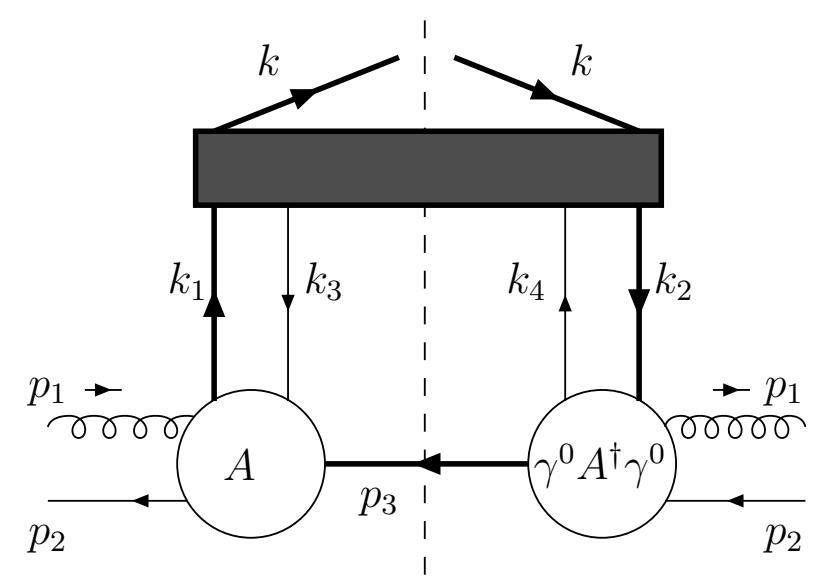

Fig.2

Fig.2. Diagram representation for Eq.(5). In the figure $k_{4}=k_{1}-k_{2}+k_{3}$, the dash line is the cut, the thick lines below the black box are for heavy quarks.

The factorization can be performed by decomposing the color- and Dirac indices of the matrix element. The decomposition of color indices of the matrix element can be performed in a straightforward way and one can identify that the $Q \bar{q}$ pair is in a color-singlet or a color-octet. We denote the color-singlet- and color-octet contribution as $d \sigma_{R}^{(1)}$ and $d \sigma_{R}^{(8)}$ respectively and 


$$
d \sigma_{R}=d \sigma_{R}^{(1)}+d \sigma_{R}^{(8)}
$$

The contributions read:

$$
\begin{aligned}
d \sigma_{R}^{(1)}= & \frac{1}{2 \hat{s}} \frac{d^{3} k}{(2 \pi)^{3} 2 k^{0}} \int \frac{d^{3} p_{3}}{(2 \pi)^{3} 2 p_{3}^{0}} \cdot \int \frac{d^{4} k_{1}}{(2 \pi)^{4}} \frac{d^{4} k_{2}}{(2 \pi)^{4}} \\
& \cdot \frac{1}{9} \operatorname{Tr} A_{i j}\left(p_{1}, p_{2}, k_{1}, p_{3}\right) \operatorname{Tr}\left(\gamma^{0} A^{\dagger}\left(p_{1}, p_{2}, k_{2}, p_{3}\right) \gamma^{0}\right)_{k l} \int d^{4} x_{1} d^{4} x_{2} d^{4} x_{3} \\
& \cdot e^{-i k_{1} \cdot x_{1}+i k_{2} \cdot x_{2}-i x_{3} \cdot k_{3}}\left\langle 0\left|\bar{q}_{k}(0) Q_{l}\left(x_{2}\right) a_{H_{Q}}^{\dagger}(k) a_{H_{Q}}(k) \bar{Q}_{i}\left(x_{1}\right) q_{j}\left(x_{3}\right)\right| 0\right\rangle, \\
d \sigma_{R}^{(8)}= & \frac{1}{2 \hat{s}} \frac{d^{3} k}{(2 \pi)^{3} 2 k^{0}} \int \frac{d^{3} p_{3}}{(2 \pi)^{3} 2 p_{3}^{0}} \cdot \int \frac{d^{4} k_{1}}{(2 \pi)^{4}} \frac{d^{4} k_{2}}{(2 \pi)^{4}} \\
& \cdot \frac{1}{2} \operatorname{Tr} T^{b} A_{i j}\left(p_{1}, p_{2}, k_{1}, p_{3}\right) \operatorname{Tr}\left(T^{b} \gamma^{0} A^{\dagger}\left(p_{1}, p_{2}, k_{2}, p_{3}\right) \gamma^{0}\right)_{k l} \int d^{4} x_{1} d^{4} x_{2} d^{4} x_{3} \\
& \cdot e^{-i k_{1} \cdot x_{1}+i k_{2} \cdot x_{2}-i x_{3} \cdot k_{3}\left\langle 0\left|\bar{q}_{k}(0) T^{a} Q_{l}\left(x_{2}\right) a_{H_{Q}}^{\dagger}(k) a_{H_{Q}}(k) \bar{Q}_{i}\left(x_{1}\right) T^{a} q_{j}\left(x_{3}\right)\right| 0\right\rangle .}
\end{aligned}
$$

In the above equations the traces are taken over color indices and the indices $i, j, k, l$ stand only for Dirac indices.

In this section we will consider the case where the transverse momentum $k_{t}$ of the produced hadron is very large, and all quark masses can be neglected. To evaluate the contributions we take a frame in which the $z$-direction is the moving direction of $H_{Q}$ and define a light-cone coordinate system in which a vector $V$ is expressed with components $V^{\mu}(\mu=0,1,2,3)$ in usual coordinate system as:

$$
V^{\mu}=\left(V^{+}, V^{-}, V^{1}, V^{2}\right)=\left(\frac{V^{0}+V^{3}}{\sqrt{2}}, \frac{V^{0}-V^{3}}{\sqrt{2}}, V^{1}, V^{2}\right)
$$

We also introduce two light-cone vectors:

$$
n^{\mu}=(0,1,0,0), \quad l^{\mu}=(1,0,0,0)
$$

The momentum $k$ reads:

$$
k^{\mu}=\left(k^{+}, k^{-}, 0,0\right)=\left(k^{+}, \frac{M_{H_{Q}}^{2}}{2 k^{+}}, 0,0\right) \approx\left(k^{+}, 0,0,0\right)=k^{+} l^{\mu} .
$$

The $x$-dependence of the matrix elements in Eq.(7) and Eq.(8) is controlled by different scales. The $x^{-}$dependence is controlled by the large scale $k^{+}$, while the $x^{+}$-dependence is controlled by the small scale $k^{-}$or $\Lambda_{Q C D}$, the $x^{1}$ - and $x^{2}$-dependence is controlled by $\Lambda_{Q C D}$. Therefore, as an approximation one can neglect the dependence of $x^{-}, x^{1}$ and $x^{2}$. Corrections to this can be systematically added. This results in the so called collinear expansion. The leading order of the expansion is to set

$$
k_{i}^{\mu}=\left(z_{i} k^{+}, 0,0,0\right)=z_{i} k^{+} l^{\mu}, \quad \text { for } i=1,2,3,
$$


in the amplitude $A$ and $A^{\dagger}$. Taking the color-singlet in Eq.(7) as an example, we obtain:

$$
\begin{aligned}
d \sigma_{R}^{(1)}= & \frac{1}{2 \hat{s}} \frac{d^{3} k}{(2 \pi)^{3} 2 k^{0}} \int \frac{d^{3} p_{3}}{(2 \pi)^{3} 2 p_{3}^{0}} \cdot \int \frac{d z_{1}}{(2 \pi)} \frac{d z_{2}}{(2 \pi)}\left(k^{+}\right)^{2}(2 \pi)^{3} \delta_{-}^{3}\left(p_{1}+p_{2}-k_{1}-p_{3}\right) \\
& \frac{1}{9} \operatorname{Tr} A_{i j}\left(p_{1}, p_{2}, z_{1} k^{+} l, p_{3}\right) \operatorname{Tr}\left(\gamma^{0} A^{\dagger}\left(p_{1}, p_{2}, z_{2} k^{+} l, p_{3}\right) \gamma^{0}\right)_{k l} \\
& \cdot \int d x_{1}^{-} d x_{2}^{-} d x_{3}^{-} e^{-i k^{+}\left(z_{1} x_{1}^{-}-z_{2} x_{2}^{-}+z_{3} x_{3}^{-}\right)} \\
& \left\langle 0\left|\bar{q}_{k}(0) Q_{l}\left(x_{2}^{-} n\right) a_{H_{Q}}^{\dagger}(k) a_{H_{Q}}(k) \bar{Q}_{i}\left(x_{1}^{-} n\right) q_{j}\left(x_{3}^{-} n\right)\right| 0\right\rangle,
\end{aligned}
$$

where $\delta_{-}^{3}(q)=\delta\left(q^{-}\right) \delta\left(q^{1}\right) \delta\left(q^{2}\right)$. With Eq. (12) the variable $z_{i}^{-1}(i=1,2,3)$ is just the momentum fraction of partons carried by $H_{Q}$. Because the momentum conservation they are bounded, for example, $z_{i}>1$ for $i=1,2,3$. Now the decomposition of the Dirac indices can be performed. We will only keep the leading terms, i.e., the twist-4 terms. The decomposition read:

$$
\begin{aligned}
& k^{+} \int \frac{d x_{1}^{-}}{(2 \pi)} \frac{d x_{2}^{-}}{(2 \pi)} \frac{d x_{3}^{-}}{(2 \pi)} e^{-i k^{+}\left(z_{1} x_{1}^{-}-z_{2} x_{2}^{-}+z_{3} x_{3}^{-}\right)}\left\langle 0\left|\bar{q}_{k}(0) Q_{l}\left(x_{2}^{-} n\right) a_{H_{Q}}^{\dagger}(k) a_{H_{Q}}(k) \bar{Q}_{i}\left(x_{1}^{-} n\right) q_{j}\left(x_{3}^{-} n\right)\right| 0\right\rangle \\
= & \left(\gamma^{-}\right)_{j i}\left(\gamma^{-}\right)_{l k} \mathcal{T}_{1}^{(1)}\left(z_{1}, z_{2}, z_{3}\right)+\left(\gamma^{-} \gamma_{5}\right)_{j i}\left(\gamma^{-} \gamma_{5}\right)_{l k} \mathcal{T}_{2}^{(1)}\left(z_{1}, z_{2}, z_{3}\right) \\
& +\left(i \sigma^{-m}\right)_{j i}\left(i \sigma^{-m}\right)_{l k} \mathcal{T}_{3}^{(1)}\left(z_{1}, z_{2}, z_{3}\right)+\cdots,
\end{aligned}
$$

where $\cdots$ denotes the terms with twist higher than 4 . The sum over the index $m$ runs from 1 to 2. The functions $\mathcal{T}_{i}(i=1,2,3)$ are defined as:

$$
\begin{aligned}
\mathcal{T}_{1}^{(1)}\left(z_{1}, z_{2}, z_{3}\right)= & \frac{k^{+}}{16} \int \frac{d x_{1}^{-}}{2 \pi} \frac{d x_{2}^{-}}{2 \pi} \frac{d x_{3}^{-}}{2 \pi} e^{-i k^{+}\left(z_{1} x_{1}^{-}-z_{2} x_{2}^{-}+z_{3} x_{3}^{-}\right)} \\
\cdot & \left\langle 0\left|\bar{q}(0) \gamma^{+} Q\left(x_{2}^{-} n\right) a_{H_{Q}}^{\dagger} a_{H_{Q}} \bar{Q}\left(x_{1}^{-} n\right) \gamma^{+} q\left(x_{3}^{-} n\right)\right| 0\right\rangle, \\
\mathcal{T}_{2}^{(1)}\left(z_{1}, z_{2}, z_{3}\right)= & \frac{k^{+}}{16} \int \frac{d x_{1}^{-}}{2 \pi} \frac{d x_{2}^{-}}{2 \pi} \frac{d x_{3}^{-}}{2 \pi} e^{-i k^{+}\left(z_{1} x_{1}^{-}-z_{2} x_{2}^{-}+z_{3} x_{3}^{-}\right)} \\
& \cdot\left\langle 0\left|\bar{q}(0) \gamma^{+} \gamma_{5} Q\left(x_{2}^{-} n\right) a_{H_{Q}}^{\dagger} a_{H_{Q}} \bar{Q}\left(x_{1}^{-} n\right) \gamma^{+} \gamma_{5} q\left(x_{3}^{-} n\right)\right| 0\right\rangle, \\
\mathcal{T}_{3}^{(1)}\left(z_{1}, z_{2}, z_{3}\right)= & \frac{k^{+}}{16} \int \frac{d x_{1}^{-}}{2 \pi} \frac{d x_{2}^{-}}{2 \pi} \frac{d x_{3}^{-}}{2 \pi} e^{-i k^{+}\left(z_{1} x_{1}^{-}-z_{2} x_{2}^{-}+z_{3} x_{3}^{-}\right)} \\
& \cdot\left\langle 0\left|\bar{q}(0) i \sigma^{+i} Q\left(x_{2}^{-} n\right) a_{H_{Q}}^{\dagger} a_{H_{Q}} \bar{Q}\left(x_{1}^{-} n\right) i \sigma^{+i} q\left(x_{3}^{-} n\right)\right| 0\right\rangle,
\end{aligned}
$$

these three functions represent the nonperturbative effects related to the produced hadron. They are invariant under a Lorentz boost along the moving direction of $H_{Q}$. The dimension of these functions are 2 in mass, hence they are proportional to $\Lambda_{Q C D}^{2}$. With these results we obtain

$$
\begin{aligned}
d \sigma_{R}^{(1)}= & \frac{1}{2 \hat{s}} \frac{d^{3} k}{(2 \pi)^{2} 2 k^{0}} \int \frac{d^{3} p_{3}}{(2 \pi)^{3} 2 p_{3}^{0}} \cdot \int \frac{d z_{1}}{z_{1}} \frac{d z_{2}}{z_{2}}(2 \pi)^{3} \delta_{-}^{3}\left(p_{1}+p_{2}-k_{1}-p_{3}\right) \\
& \cdot \frac{1}{9 k^{+}}\left\{\operatorname{Tr}\left(\gamma \cdot k_{1} A\left(p_{1}, p_{2}, k_{1}, p_{3}\right)\right) \operatorname{Tr}\left(\gamma \cdot k_{2} \gamma^{0} A^{\dagger}\left(p_{1}, p_{2}, k_{2}, p_{3}\right) \gamma^{0}\right) \cdot \mathcal{T}_{1}^{(1)}\left(z_{1}, z_{2}, z_{3}\right)\right. \\
& +\operatorname{Tr}\left(\gamma \cdot k_{1} \gamma_{5} A\left(p_{1}, p_{2}, k_{1}, p_{3}\right)\right) \operatorname{Tr}\left(\gamma \cdot k_{2} \gamma_{5} \gamma^{0} A^{\dagger}\left(p_{1}, p_{2}, k_{2}, p_{3}\right) \gamma^{0}\right) \cdot \mathcal{T}_{2}^{(1)}\left(z_{1}, z_{2}, z_{3}\right) \\
& \left.+\operatorname{Tr}\left(i \sigma^{\mu i} k_{1 \mu} A\left(p_{1}, p_{2}, k_{1}, p_{3}\right)\right) \operatorname{Tr}\left(i \sigma^{\mu i} k_{2 \mu} \gamma^{0} A^{\dagger}\left(p_{1}, p_{2}, k_{2}, p_{3}\right) \gamma^{0}\right) \cdot \mathcal{T}_{3}^{(1)}\left(z_{1}, z_{2}, z_{3}\right)\right\},
\end{aligned}
$$


for the color-octet contributions we obtain:

$$
\begin{aligned}
d \sigma_{R}^{(8)}= & \frac{1}{2 \hat{s}} \frac{d^{3} k}{(2 \pi)^{2} 2 k^{0}} \int \frac{d^{3} p_{3}}{(2 \pi)^{3} 2 p_{3}^{0}} \cdot \int \frac{d z_{1}}{z_{1}} \frac{d z_{2}}{z_{2}}(2 \pi)^{3} \delta_{-}^{3}\left(p_{1}+p_{2}-k_{1}-p_{3}\right) \\
& \cdot \frac{1}{2 k^{+}}\left\{\operatorname{Tr}\left(\gamma \cdot k_{1} T^{a} A\left(p_{1}, p_{2}, k_{1}, p_{3}\right)\right) \operatorname{Tr}\left(\gamma \cdot k_{2} \gamma^{0} T^{a} A^{\dagger}\left(p_{1}, p_{2}, k_{2}, p_{3}\right) \gamma^{0}\right) \cdot \mathcal{T}_{1}^{(8)}\left(z_{1}, z_{2}, z_{3}\right)\right. \\
& +\operatorname{Tr}\left(\gamma \cdot k_{1} \gamma_{5} T^{a} A\left(p_{1}, p_{2}, k_{1}, p_{3}\right)\right) \operatorname{Tr}\left(\gamma \cdot k_{2} \gamma_{5} \gamma^{0} T^{a} A^{\dagger}\left(p_{1}, p_{2}, k_{2}, p_{3}\right) \gamma^{0}\right) \cdot \mathcal{T}_{2}^{(8)}\left(z_{1}, z_{2}, z_{3}\right) \\
& \left.+\operatorname{Tr}\left(i \sigma^{\mu i} k_{1 \mu} T^{a} A\left(p_{1}, p_{2}, k_{1}, p_{3}\right)\right) \operatorname{Tr}\left(i \sigma^{\mu i} k_{2 \mu} \gamma^{0} T^{a} A^{\dagger}\left(p_{1}, p_{2}, k_{2}, p_{3}\right) \gamma^{0}\right) \cdot \mathcal{T}_{3}^{(8)}\left(z_{1}, z_{2}, z_{3}\right)\right\}
\end{aligned}
$$

with $k_{i}=z_{i} k^{+} l$. The definitions for the functions $\mathcal{T}_{i}^{(8)}\left(z_{1}, z_{2}, z_{3}\right)(i=1,2,3)$ can be obtained by replacing the color matrix $1 \otimes 1$ with $T^{a} \otimes T^{a}$ in the definitions for $\mathcal{T}_{i}^{(1)}\left(z_{1}, z_{2}, z_{3}\right)(i=1,2,3)$, respectively.

The integration over phase space can be easily performed in the center-of-mass frame. Consider the integral

$$
\int \frac{d^{3} k}{(2 \pi)^{3} 2 k^{0}} \int \frac{d^{3} p_{3}}{(2 \pi)^{3} 2 p_{3}^{0}} \cdot(2 \pi)^{3} \delta_{-}^{3}\left(p_{1}+p_{2}-k_{1}-p_{3}\right) \cdot \frac{1}{k^{+}}
$$

because the only nonzero component of $k_{1}$ is $k_{1}^{+}, k_{1}$ can be set zero in the $\delta_{-}$function. The integration over $p_{3}$ can be done with the $\delta_{-}$function. We insert in the above integral the identity:

$$
\int d z_{3} k^{+} \delta\left(p_{1}^{+}+p_{+}-z_{1} k^{+}-z_{3} k^{+}\right)=1=\int d z_{3} \sqrt{2}|\mathbf{k}| \delta\left(\frac{\sqrt{\hat{s}}}{\sqrt{2}}-\sqrt{2}\left(z_{1}+z_{3}\right)|\mathbf{k}|\right),
$$

and obtain the phase space integral as

$$
\int \frac{d^{3} k}{(2 \pi)^{3} 2 k^{0}} \int \frac{d^{3} p_{3}}{(2 \pi)^{3} 2 p_{3}^{0}} \cdot(2 \pi)^{3} \delta_{-}^{3}\left(p_{1}+p_{2}-k_{1}-p_{3}\right) \cdot \frac{1}{k^{+}}=\frac{1}{4(2 \pi)^{2} \hat{s}} \int d \hat{t} \int d z_{3} \cdot \frac{1}{\left(z_{1}+z_{3}\right)^{2}} .
$$

With this we can express our results for $d \sigma / d \hat{t}$. The remainder of the calculations are straightforward. Without detailed calculation one can find that the coefficient of $\mathcal{T}_{3}^{(i)}$ is zero, the reason is that we consider unpolarized hadron beams here and the polarizations of initial partons are averaged. Due to helicity conservation, the coefficient must be zero. If we consider polarized hadron beams, $\mathcal{T}_{3}^{(i)}$ can lead to a nonzero contribution. With the structure of $\mathcal{T}_{1}^{(i)}$ and $\mathcal{T}_{2}^{(i)}$ one can also find that the coefficients of $\mathcal{T}_{1}^{(i)}$ and $\mathcal{T}_{2}^{(i)}$ are same. Finally we have:

$$
\begin{aligned}
\frac{d \sigma_{R}^{(1)}}{d \hat{t}}(\hat{s}, \hat{t})= & \frac{2 \pi \alpha_{s}^{3} 1}{243 \hat{s}^{2}} \int \frac{d z_{1}}{z_{1}} \frac{d z_{2}}{z_{2}} \frac{d z_{3}}{z_{3}} \frac{1}{\left(z_{1}+z_{3}\right)^{2}} \frac{\left\{\mathcal{T}_{1}^{(1)}+\mathcal{T}_{2}^{(1)}\right\}}{\left(z_{1}-z_{2}+z_{3}\right)(\hat{s}+\hat{t})} \\
& \left\{\frac{\hat{s}^{2}}{\hat{t}^{2}}\left(9 z_{1}+z_{3}\right)\left(z_{1}+8 z_{2}+z_{3}\right)+\frac{\hat{s}}{\hat{t}}\left[27 z_{1}^{2}+297 z_{1} z_{2}+29 z_{1} z_{3}+25 z_{2} z_{3}+2 z_{3}^{2}\right]\right. \\
& +\frac{4 \hat{t}^{2}}{\hat{s}^{2}}\left[81 z_{3}\left(z_{3}-z_{2}\right)+z_{1}\left(25 z_{2}+81 z_{3}\right)\right]-\frac{4 \hat{t}}{\hat{s}}\left[81 z_{1}^{2}+9 z_{3}\left(8 z_{2}+z_{3}\right)+z_{1}\left(90 z_{3}-86 z_{2}\right)\right] \\
& \left.-144 z_{1}^{2}+469 z_{1} z_{2}-179 z_{1} z_{3}-109 z_{2} z_{3}-35 z_{3}^{2}\right\}
\end{aligned}
$$




$$
\begin{aligned}
\frac{d \sigma_{R}^{(8)}}{d \hat{t}}(\hat{s}, \hat{t})= & \frac{\pi \alpha_{s}^{3}}{162 \hat{s}^{2}} \int \frac{d z_{1}}{z_{1}} \frac{d z_{2}}{z_{2}} \frac{d z_{3}}{z_{3}} \frac{1}{\left(z_{1}+z_{3}\right)^{2}} \frac{\left\{\mathcal{T}_{1}^{(8)}+\mathcal{T}_{2}^{(8)}\right\}}{\left(z_{1}-z_{2}+z_{3}\right)[\hat{s}+\hat{t}]} \\
& \left\{\frac{\hat{s}^{2}}{\hat{t}^{2}}\left[63 z_{1}^{2}+z_{3}\left(z_{2}+62 z_{3}\right)+z_{1}\left(9 z_{2}+125 z_{3}\right)\right]\right. \\
& +\frac{\hat{s}}{\hat{t}}\left[207 z_{1}^{2}+99 z_{1} z_{2}+331 z_{1} z_{3}+83 z_{2} z_{3}+124 z_{3}^{2}\right] \\
& +\frac{7 \hat{t}^{2}}{\hat{s}^{2}}\left[45 z_{3}\left(z_{3}-z_{2}\right)+z_{1}\left(41 z_{2}+45 z_{3}\right)\right]-\frac{4 \hat{t}}{\hat{s}}\left[81 z_{1}^{2}+9 z_{3}\left(z_{2}+8 z_{3}\right)+z_{1}\left(153 z_{3}-148 z_{2}\right)\right] \\
& \left.-18 z_{1}^{2}+5 z_{1}\left(79 z_{2}-47 z_{3}\right)+z_{3}\left(199 z_{2}-217 z_{3}\right)\right\} .
\end{aligned}
$$

Similarly for the process

$$
\gamma\left(p_{1}\right)+\bar{q}\left(p_{2}\right) \rightarrow H_{Q}(k)+X
$$

we have

$$
\begin{aligned}
\frac{d \tilde{\sigma}_{R}^{(i)}}{d \hat{t}}(\hat{s}, \hat{t})= & \frac{\pi \alpha_{s}^{2} \alpha}{3 \hat{s}^{2}} \int d \hat{t} \int \frac{d z_{1}}{z_{1}} \frac{d z_{2}}{z_{2}} \frac{d z_{3}}{z_{3}} \frac{1}{\left(z_{1}+z_{3}\right)^{2}} \frac{\mathcal{C}^{(i)} e_{Q}^{2}\left\{\mathcal{T}_{1}^{(i)}+\mathcal{T}_{2}^{(i)}\right\}}{\left(z_{1}-z_{2}+z_{3}\right)} \\
& \left\{\frac{\left[\hat{t}^{2} z_{1} z_{2}+\hat{s}^{2} z_{3}\left(z_{1}-z_{2}+z_{3}\right)\right]}{\hat{t}^{2}(\hat{s}+\hat{t})}\left[1+\kappa \frac{\hat{t}}{\hat{s}}\right]^{2}\right\}
\end{aligned}
$$

where $e_{Q}\left(e_{q}\right)$ is the charge of the heavy(light) quark and

$$
\kappa=\frac{e_{q}}{e_{Q}}, \quad \mathcal{C}^{(1)}=\frac{256}{27}, \quad \mathcal{C}^{(8)}=\frac{8}{9} .
$$

With these results one can predict the leading particle effect in hadron collision and photoproduction. We denote the anti-particle of $H_{Q}$ as $\bar{H}_{Q}$. Because of the symmetry of charge conjugation, the differential cross section for $g\left(p_{1}\right)+q\left(p_{2}\right) \rightarrow \bar{H}_{Q}(k)+X$ is the same as given above. The leading particle effect is generated by the asymmetry between the distributions of $q$ and $\bar{q}$. We consider the production in the hadron collision:

$$
\begin{aligned}
& A\left(P_{1}\right)+B\left(P_{2}\right) \rightarrow H_{Q}(k)+X, \\
& A\left(P_{1}\right)+B\left(P_{2}\right) \rightarrow \bar{H}_{Q}(k)+X,
\end{aligned}
$$

where $A$ and $B$ are the initial hadrons, whose spin is not observed. We define the variables:

$$
s=\left(P_{1}+P_{2}\right)^{2}, \quad t=\left(P_{2}-k\right)^{2}, \quad u=\left(P_{1}-k\right)^{2} .
$$

The leading particle effect can be predicted as:

$$
\begin{aligned}
\frac{d \sigma}{d t}\left(H_{Q}\right)-\frac{d \sigma}{d t}\left(\bar{H}_{Q}\right) & =\int d x_{1} d x_{2} f_{g / A}\left(x_{1}\right)\left[f_{\bar{q} / B}\left(x_{2}\right)-f_{q / B}\left(x_{2}\right)\right] \cdot \frac{d \sigma_{R}}{d \hat{t}}\left(\hat{s}=x_{1} x_{2} s, \hat{t}=x_{2} t\right) \\
& +\int d x_{1} d x_{2} f_{g / B}\left(x_{2}\right)\left[f_{\bar{q} / A}\left(x_{1}\right)-f_{q / A}\left(x_{1}\right)\right] \cdot \frac{d \sigma_{R}}{d \hat{t}}\left(\hat{s}=x_{1} x_{2} s, \hat{t}=x_{1} u\right),
\end{aligned}
$$


where $d \sigma_{R}$ is the sum of contributions given in Eq.(21) and Eq.(22), $f_{a / A}(x)$ is the distribution for parton $a$ in hadron $A$. Similarly, one can also obtain the leading particle effect in photoproduction, replacing $A$ with a photon in Eq.(26) we obtain:

$$
\frac{d \sigma}{d t}\left(H_{Q}\right)-\frac{d \sigma}{d t}\left(\bar{H}_{Q}\right)=\int d x_{1}\left[f_{\bar{q} / B}\left(x_{1}\right)-f_{q / B}\left(x_{1}\right)\right] \cdot \frac{d \tilde{\sigma}_{R}}{d \hat{t}}\left(\hat{s}=x_{1} s, \hat{t}=x_{1} t\right)
$$

where $d \tilde{\sigma}_{R}=d \tilde{\sigma}_{R}^{(1)}+d \tilde{\sigma}_{R}^{(8)}$. If $A$ is the anti-particle of $B$, then the leading particle effect vanishes because of the charge conjugation symmetry. Since the nonperturbative functions $\mathcal{T}_{i}^{(1)}$ and $\mathcal{T}_{i}^{(8)}$ for $i=1,2$ are unknown, numerical predictions can not be made. These functions may be studied with nonperturbative methods or extracted from experimental results, they are universal like parton distributions. Once they are known, one can make numerical predictions. Our results here hold for the case with large transverse momentum of the produced hadron, i.e. For $s \rightarrow \infty$ and $t \rightarrow \infty$, but with the ratio $t / s$ being fixed, one obtains the power behavior

$$
\frac{d \sigma}{d t}\left(H_{Q}\right)-\frac{d \sigma}{d t}\left(\bar{H}_{Q}\right) \sim \frac{1}{s^{3}}
$$

in comparison with the production at twist-2 level

$$
\frac{d \sigma}{d t}\left(H_{Q}\right) \sim \frac{d \sigma}{d t}\left(\bar{H}_{Q}\right) \sim \frac{1}{s^{2}}
$$

hence, the leading particle effect through quark recombination is suppressed in comparison of the production rate by a factor $s^{-1}$, which is typical for twist-4 effects.

In [3] contributions from quark recombination to inclusive $\pi$ production is studied, partonic processes like $g \bar{q} \rightarrow \pi g$ and $g q \rightarrow q \pi$ are calculated by taking a wave function at leading twist for $\pi$. This is different than our approach. If one only keeps the contribution from $\mathcal{T}_{2}^{(1)}$ in Eq.(21) and neglects all other contributions, and then for $\mathcal{T}_{2}^{(1)}$ one uses the approximation of vacuum saturation to write it in term of the $\pi$ wave function at leading twist, our approach is equivalent to that in [3]. It is clear that there are no reasons to neglect other contributions and to use the approximation of vacuum saturation. In [3] the results are given by taking asymptotic form of the wave function, where the integration over the momentum fraction as the variable of the wave function is performed analytically. This prevents us from a direct comparison with our results. From our results it is clear that the effect of quark recombination is characterized by 2 nonperturbative functions, effectively for unpolarized beams, while for polarized beams there are in general 6 nonperturbative functions.

\section{HQET Factorization for Quark Recombination}

In this section we will use nonrelativistic normalization for $H_{Q}$ and heavy quarks $Q$. If the pole mass $m_{Q}$ of the heavy quark $Q$ is heavy enough, one can expand the heavy quark field $Q(x)$ with the field in HQET [22]:

$$
\begin{aligned}
& Q(x)=e^{-i m_{Q} v \cdot x} \cdot\left\{h(x)+\mathcal{O}\left(m_{Q}^{-1}\right)\right\}+\cdots, \\
& \bar{Q}(x)=e^{+i m_{Q} v \cdot x} \cdot\left\{\bar{h}(x)+\mathcal{O}\left(m_{Q}^{-1}\right)\right\}+\cdots,
\end{aligned}
$$


where $v$ is the four velocity of $H_{Q}$, i.e., $v=k / M_{H_{Q}} . h(x)$ is the field of HQET and depends on $v$ implicitly, the $\cdots$ denotes the part of anti-quark. The fields have the following property:

$$
v \cdot \gamma h(x)=h(x), \quad \bar{h}(x) v \cdot \gamma=\bar{h}(x) .
$$

For $m_{Q} \rightarrow \infty$ the most momentum of a produced $Q$ is carried by $H_{Q}$, where the produced heavy quark $Q$ combines with other light quarks and gluons to form the hadron $H_{Q}$. Using this fact and HQET one can expand the cross-section for production of $H_{Q}$ in $\Lambda_{Q C D} / m_{Q}$. Following the proposed factorization in [23,24], the production rate at leading order of $\Lambda_{Q C D} / m_{Q}$ is a product of the production rate of $Q$ with a matrix element defined in HQET, the momentum of $H_{Q}$ is approximated by the momentum of $Q$ and $M_{H_{Q}} \approx m_{Q}$. For example, the production of $H_{Q}$ via gluon fusion, i.e., $g+g \rightarrow H_{Q}+X$, the differential cross-section can be written as:

$$
d \sigma\left(g+g \rightarrow H_{Q}+X\right)=d \sigma(g+g \rightarrow Q+X) \cdot \frac{1}{6}\left\langle 0\left|\operatorname{Tr} h a_{H_{Q}}^{\dagger} a_{H_{Q}} \bar{h}\right| 0\right\rangle \cdot \cdot\left\{1+\mathcal{O}\left(\frac{\Lambda_{Q C D}^{2}}{m_{Q}^{2}}\right)\right\}
$$

where matrix element is defined in the rest frame of $H_{Q}$. The above equation has a close correspondence to inclusive decay of $H_{Q}$ with the factorization, the corresponding matrix element for inclusive decay of $H_{Q}$ equals 1 because flavor conservation of HQET, it leads to the well known result $[22]$ :

$$
\Gamma\left(H_{Q} \rightarrow X\right)=\Gamma(Q \rightarrow X) \cdot\left\{1+\mathcal{O}\left(\frac{\Lambda_{Q C D}^{2}}{m_{Q}^{2}}\right)\right\}
$$

At the next-to-leading order for the decay width the nonperturbative effect is represented by dimension 5 operators, corresponding operators for production can also be found. These operators are bilinear in quark fields. Going beyond this order, i.e., at the order of $m_{Q}^{-3}$, one will encounter four quark operators, these 4-quark operators represent the nonperturbative effect for quark recombination.

For the contributions from quark recombination, we neglect higher orders in Eq.(32). The color-singlet contribution reads:

$$
\begin{aligned}
d \sigma_{R}^{(1)}= & \frac{1}{2 \hat{s}} \frac{d^{3} k}{(2 \pi)^{3}} \int \frac{d^{3} p_{3}}{(2 \pi)^{3}} \cdot \int \frac{d^{4} k_{1}}{(2 \pi)^{4}} \frac{d^{4} k_{2}}{(2 \pi)^{4}} \frac{1}{9} \operatorname{Tr} A_{i j}\left(p_{1}, p_{2}, k_{1}, p_{3}\right) \operatorname{Tr}\left(\gamma^{0} A^{\dagger}\left(p_{1}, p_{2}, k_{2}, p_{3}\right) \gamma^{0}\right)_{k l} \\
& \cdot \int d^{4} x_{1} d^{4} x_{2} d^{4} x_{3} e^{-i k_{1} \cdot x_{1}+i k_{2} \cdot x_{2}-i x_{3} \cdot k_{3}-i m_{Q} v \cdot x_{2}+i m_{Q} v \cdot x_{1}} \\
& \cdot\left\langle 0\left|\bar{q}_{k}(0) h_{l}\left(x_{2}\right) a_{H_{Q}}^{\dagger}(k) a_{H_{Q}}(k) \bar{h}_{i}\left(x_{1}\right) q_{j}\left(x_{3}\right)\right| 0\right\rangle .
\end{aligned}
$$

Now the space-time dependence of the matrix element is controlled by the small scale $\Lambda_{Q C D}$, reflecting the fact that the most momentum of $Q$ is carried by the hadron $H_{Q}$, the difference between the momentum of $Q$ and that of $H_{Q}$ is order of $\Lambda_{Q C D}$. At first look, one may neglect the space-time dependence as an approximation, used in deriving Eq.(34) or Eq.(35). This implies that the light antiquark $\bar{q}$ will have zero momentum after scattering. It results in that the amplitude $A$ will be divergent because the gluon propagator attached to $\bar{q}$ in Fig.1. To have meaningful predictions one can not neglect the space-time dependence here. The momentum of $\bar{q}$ is of order 
of $\Lambda_{Q C D}$, one can expand the amplitude in this momentum, this approach is used in [18-20]. We will use the approach here to regularize the divergence. For doing this we write the variables $x_{i}$ as:

$$
x_{i}^{\mu}=v \cdot x_{i} v^{\mu}+x_{T i}^{\mu}=\omega_{i} v^{\mu}+x_{T i}^{\mu}, \quad \text { for } i=1,2,3,
$$

momenta can also be decomposed similarly as above. The dependence of the matrix element on $x_{T i}(i=1,2,3)$ can be safely neglected, and we have:

$$
\begin{aligned}
d \sigma_{R}^{(1)}= & \frac{1}{2 \hat{s}} \frac{d^{3} k}{(2 \pi)^{3}} \int \frac{d^{3} p_{3}}{(2 \pi)^{3}}(2 \pi)^{3} \delta^{3}\left(p_{T 1}+p_{T 2}-p_{T 3}\right) \\
& \cdot \int \frac{d \eta_{1}}{2 \pi} \frac{d \eta_{2}}{2 \pi} \frac{1}{9} \operatorname{Tr} A_{i j}\left(p_{1}, p_{2}, k_{1}, p_{3}\right) \operatorname{Tr}\left(\gamma^{0} A^{\dagger}\left(p_{1}, p_{2}, k_{2}, p_{3}\right) \gamma^{0}\right)_{k l} \\
& \cdot \int d \omega_{1} d \omega_{2} d \omega_{3} e^{-i \eta_{1} \cdot \omega_{1}+i \eta_{2} \cdot \omega_{2}-i \eta_{3} \cdot \omega_{3}}\left\langle 0\left|\bar{q}_{k}(0) h_{l}\left(\omega_{2} v\right) a_{H_{Q}}^{\dagger}(k) a_{H_{Q}}(k) \bar{h}_{i}\left(\omega_{1} v\right) q_{j}\left(\omega_{3} v\right)\right| 0\right\rangle,
\end{aligned}
$$

with

$$
k_{1}=\left(m_{Q}+\eta_{1}\right) v, \quad k_{2}=\left(m_{Q}+\eta_{2}\right) v, \quad k_{3}=\eta_{3} v
$$

To separate the divergences more clearly, we use translational invariance for the matrix element to shift the variable of fields with $-\omega_{2} v$, and rearrange the variables as: $\omega_{2} \rightarrow-\omega_{2}, \omega_{3}-\omega_{2} \rightarrow \omega_{1}$, $\omega_{1}-\omega_{2} \rightarrow \omega_{3}$, and $\eta_{1}+\eta_{3}-\eta_{2} \rightarrow \eta_{2}, \eta_{1} \rightarrow \eta_{3}, \eta_{3} \rightarrow \eta_{1}$. After this re-arrangement the momentum $k_{i}$ reads:

$$
k_{1}=\left(m_{Q}+\eta_{3}\right) v, \quad k_{2}=\left(m_{Q}+\left(\eta_{3}+\eta_{1}-\eta_{2}\right)\right) v, \quad k_{3}=\eta_{1} v .
$$

The light quark $\bar{q}$ carries the momentum $\eta_{1} v$ and $\eta_{2} v$ in the amplitude $A$ and $A^{\dagger}$ respectively. Now we observe that for $\eta_{3} \rightarrow 0$ the amplitude $A$ and $A^{\dagger}$ is finite, while for $\eta_{1} \rightarrow 0$ and $\eta_{2} \rightarrow 0$ they are divergent respectively. We expand the amplitude $A$ and $A^{\dagger}$ respectively in $\eta_{1} v$ and $\eta_{2} v$ and only keep the leading terms in the expansion in $\eta_{1}, \eta_{2}$ and $\eta_{3}$, i.e.;

$$
A_{i j}=\frac{T_{i j}}{\eta_{1}}(1+\cdots), \quad A_{i j}^{\dagger}=\frac{T_{i j}^{\dagger}}{\eta_{2}}(1+\cdots),
$$

where $T_{i j}$ and $T_{i j}^{\dagger}$ do not depends on $\eta_{1}, \eta_{2}$ and $\eta_{3}$. Using Eq.(41) the integral over $\eta_{3}$ and $\omega_{3}$ can be performed and we obtain:

$$
\begin{aligned}
d \sigma_{R}^{(1)}= & \frac{1}{2 \hat{s}} \frac{d^{3} k}{(2 \pi)^{3}} \int \frac{d^{3} p_{3}}{(2 \pi)^{3}}(2 \pi)^{3} \delta^{3}\left(p_{T 1}+p_{T 2}-p_{T 3}\right) \\
& \cdot \int \frac{d \eta_{2}}{2 \pi} \frac{1}{9 \eta_{1} \eta_{2}} \operatorname{Tr} T_{i j}\left(p_{1}, p_{2}, m_{Q} v, p_{3}\right) \operatorname{Tr}\left(\gamma^{0} T^{\dagger}\left(p_{1}, p_{2}, m_{Q} v, p_{3}\right) \gamma^{0}\right)_{k l} \\
& \cdot \int d \omega_{1} d \omega_{2} e^{+i \eta_{2} \omega_{2}-i \eta_{1} \cdot \omega_{1}}\left\langle 0\left|\bar{q}_{k}\left(\omega_{2} v\right) h_{l}(0) a_{H_{Q}}^{\dagger}(k) a_{H_{Q}}(k) \bar{h}_{i}(0) q_{j}\left(\omega_{1} v\right)\right| 0\right\rangle .
\end{aligned}
$$

The Dirac indices of the Fourier transformed matrix element can be decomposed. In general there are many terms. With the property in Eq.(33) the number of terms can be reduced greatly and we have only four terms: 


$$
\begin{aligned}
& v^{0} \int \frac{d \omega_{1}}{2 \pi} \frac{d \omega_{2}}{2 \pi} e^{-i \eta_{1} \cdot \omega_{1}+i \eta_{2} \cdot \omega_{2}} \cdot\left\langle 0\left|\bar{q}_{k}\left(\omega_{2} v\right) h_{l}(0) a_{H_{Q}}^{\dagger}(k) a_{H_{Q}}(k) \bar{h}_{i}(0) q_{j}\left(\omega_{1} v\right)\right| 0\right\rangle \\
& =\left(P_{v}\right)_{j i}\left(P_{v}\right)_{l k} \mathcal{W}_{1}^{(1)}\left(\eta_{1}, \eta_{2}\right)-\left(\gamma_{5} P_{v}\right)_{j i}\left(P_{v} \gamma_{5}\right)_{l k} \mathcal{W}_{2}^{(1)}\left(\eta_{1}, \eta_{2}\right) \\
& -\left(\gamma_{T}^{\mu} P_{v}\right)_{j i}\left(P_{v} \gamma_{T \mu}\right)_{l k} \mathcal{W}_{3}^{(1)}\left(\eta_{1}, \eta_{2}\right)-\left(\gamma_{T}^{\mu} \gamma_{5} P_{v}\right)_{j i}\left(P_{v} \gamma_{T \mu} \gamma_{5}\right)_{l k} \mathcal{W}_{4}^{(1)}\left(\eta_{1}, \eta_{2}\right)
\end{aligned}
$$

where

$$
\gamma_{T}^{\mu}=\gamma^{\mu}-v \cdot \gamma v^{\mu}, \quad P_{v}=\frac{1+\gamma \cdot v}{2}
$$

and the functions are defined in the rest frame of $H_{Q}$. By using matrix elements in the rest frame the factor $v^{0}$ in Eq.(43) appears because of the nonrelativistic normalization of the state. The functions are:

$$
\begin{aligned}
& \mathcal{W}_{1}^{(1)}\left(\eta_{1}, \eta_{2}\right)=\frac{1}{4} \int \frac{d \omega_{1}}{2 \pi} \frac{d \omega_{2}}{2 \pi} e^{-i \eta_{1} \cdot \omega_{1}+i \eta_{2} \cdot \omega_{2}} \cdot\left\langle 0\left|\bar{q}\left(\omega_{2} v\right) h(0) a_{H_{Q}}^{\dagger} a_{H_{Q}} \bar{h}(0) q\left(\omega_{1} v\right)\right| 0\right\rangle, \\
& \mathcal{W}_{2}^{(1)}\left(\eta_{1}, \eta_{2}\right)=-\frac{1}{4} \int \frac{d \omega_{1}}{2 \pi} \frac{d \omega_{2}}{2 \pi} e^{-i \eta_{1} \cdot \omega_{1}+i \eta_{2} \cdot \omega_{2}} \cdot\left\langle 0\left|\bar{q}\left(\omega_{2} v\right) \gamma_{5} h(0) a_{H_{Q}}^{\dagger} a_{H_{Q}} \bar{h}(0) \gamma_{5} q\left(\omega_{1} v\right)\right| 0\right\rangle, \\
& \mathcal{W}_{3}^{(1)}\left(\eta_{1}, \eta_{2}\right)=-\frac{1}{12} \int \frac{d \omega_{1}}{2 \pi} \frac{d \omega_{2}}{2 \pi} e^{-i \eta_{1} \cdot \omega_{1}+i \eta_{2} \cdot \omega_{2}} \cdot\left\langle 0\left|\bar{q}\left(\omega_{2} v\right) \gamma_{T}^{\nu} h(0) a_{H_{Q}}^{\dagger} a_{H_{Q}} \bar{h}(0) \gamma_{T \nu} q\left(\omega_{1} v\right)\right| 0\right\rangle, \\
& \mathcal{W}_{4}^{(1)}\left(\eta_{1}, \eta_{2}\right)=-\frac{1}{12} \int \frac{d \omega_{1}}{2 \pi} \frac{d \omega_{2}}{2 \pi} e^{-i \eta_{1} \cdot \omega_{1}+i \eta_{2} \cdot \omega_{2}} \cdot\left\langle 0\left|\bar{q}\left(\omega_{2} v\right) \gamma_{T}^{\nu} \gamma_{5} h(0) a_{H_{Q}}^{\dagger} a_{H_{Q}} \bar{h}(0) \gamma_{T \nu} \gamma_{5} q\left(\omega_{1} v\right)\right| 0\right\rangle,
\end{aligned}
$$

In the above definitions the operator $a_{H_{Q}}$ creates $H_{Q}$ in its rest frame. For the color-octet matrix element one has the same decomposition as in Eq.(43) with the functions $\mathcal{W}_{i}^{(8)}\left(\eta_{1}, \eta_{2}, \eta_{3}\right)$ with $i=1, \cdots 4$. The definition of these functions are obtained by replacing the color matrix $1 \otimes 1$ with $T^{a} \otimes T^{a}$ in the above equation.

To express our results as the differential cross-section like $d \sigma / d \hat{t}$ we need to consider the phasespace integral

$$
\int \frac{d^{3} k}{(2 \pi)^{3}} \cdot \frac{d^{3} p_{3}}{(2 \pi)^{3}}(2 \pi)^{3} \delta^{3}\left(p_{T 1}+p_{T 2}-p_{T 3}\right) .
$$

Because $p_{1}+p_{2}-k_{1}-k_{3}-p_{3}$ we can insert the identity

$$
\int d \eta_{1} \delta\left(v \cdot\left(p_{1}+p_{2}-m_{Q} v-\eta_{1} v-p_{3}\right)\right)=1
$$

where we neglect $\eta_{3}$ in Eq.(40) and the momentum $k$ of $H_{Q}$ is approximated by $k=m_{Q} v+\eta_{1} v$. The $\delta$-function in Eq.(47) combining those $\delta$-functions in Eq.(46) gives usual $\delta$-functions $\delta^{4}\left(p_{1}+\right.$ $\left.p_{2}-k-p_{3}\right)$ for momentum conservation, then the phase-space integral can be calculated as usual. We obtain:

$$
\int \frac{d^{3} k}{(2 \pi)^{3}} \cdot \frac{d^{3} p_{3}}{(2 \pi)^{3}}(2 \pi)^{3} \delta^{3}\left(p_{T 1}+p_{T 2}-p_{T 3}\right) \approx \frac{m_{Q}}{8 \pi^{2} \sqrt{\hat{s}}} \int d \hat{t} d \eta_{1} v^{0}
$$


where we use $k \approx m_{Q} v$. Our results contain 8 parameters which represent nonperturbative effects. These parameters are defined as integrals with the functions $\mathcal{W}_{i}^{(1,8)}(i=1, \cdots 4)$ :

$$
w_{i}^{(1,8)}=\frac{1}{m_{Q}^{3}} \int \frac{d \eta_{1}}{\eta_{1}} \frac{d \eta_{2}}{\eta_{2}} \mathcal{W}_{i}^{(1,8)}\left(\eta_{1}, \eta_{2}\right) .
$$

These parameters are dimensionless. At first look, they may scale as $\Lambda_{Q C D}^{3} / m_{Q}^{3}$. But, the dominant contribution of the integrals comes from the region where $\eta_{1} \sim \eta_{2} \sim \Lambda_{Q C D}$, reflecting the fact that the light antiquark $\bar{q}$ carries a small fraction of the momentum of $H_{Q}$. This results in that the parameters $w_{i}^{(1,8)}(i=1,2,3,4)$ scale as $\Lambda_{Q C D} / m_{Q}$. With these parameters our results for the process $g+\bar{q} \rightarrow H_{Q}+X$ are:

$$
\frac{d \sigma_{R}^{(i)}}{d \hat{t}}(\hat{s}, \hat{t})=\frac{\pi \alpha_{s}^{3} m_{Q}^{2}}{\hat{s}^{2}} \sum_{j=1}^{4} w_{j}^{(i)} \mathcal{B}_{j}^{(i)}
$$

where

$$
\begin{gathered}
\mathcal{B}_{1}^{(1)}=\mathcal{B}_{2}^{(1)}=\frac{4}{81 \hat{s}}\left\{\frac{m_{Q}^{2}\left[127 T^{2}-16 \hat{s} T-64 \hat{s}^{2}\right]}{T^{3}}-\frac{64 U}{\hat{s}}-\frac{16 m_{Q}^{4} \hat{s}}{U T^{2}}\left(1-\frac{8 U}{T}\right)\right\} \\
\mathcal{B}_{3}^{(1)}=\mathcal{B}_{4}^{(1)}=\frac{1}{81 \hat{s}}\left\{-\frac{m_{Q}^{2}}{T}\left[19+\frac{4 T^{2}}{U^{2}}-\frac{28 T}{U}+\frac{368 U}{T}-\frac{64 U^{2}}{T^{2}}\right]\right. \\
\left.\quad-\frac{64 U}{\hat{s}}\left[1+\frac{2 U^{2}}{T^{2}}\right]-\frac{48 m_{Q}^{4} \hat{s}(8 \hat{s}+9 T)}{T^{3} U}\right\}, \\
\mathcal{B}_{1}^{(8)}=\mathcal{B}_{2}^{(8)}=\frac{2}{27 \hat{s}}\left\{\frac{m_{Q}^{4} \hat{s}\left(8 \hat{s}^{2}+8 \hat{s} T+9 T^{2}\right)}{T^{3} U^{2}}-\frac{m_{Q}^{2}}{2 T}\left[79-\frac{18 T^{2}}{U^{2}}+\frac{14 U}{T}+\frac{8 U^{2}}{T^{2}}\right]\right. \\
\left.-\frac{4 \hat{s}^{2}-\hat{s} T+4 T^{2}}{\hat{s} U}\right\}, \\
\mathcal{B}_{3}^{(8)}=\mathcal{B}_{4}^{(8)}=\frac{2}{27 \hat{s}}\left\{-\frac{U}{\hat{s}}\left[22+\frac{9 T}{U}+\frac{18 U}{T}+\frac{9 T^{2}}{U^{2}}+\frac{8 U^{2}}{T^{2}}\right]\right. \\
\left.-\frac{m_{Q}^{2}}{2 T}\left[233+\frac{266 T^{2}}{U^{2}}+\frac{316 T}{U}+\frac{10 U}{T}-\frac{8 U^{2}}{T^{2}}\right]+\frac{3 m_{Q}^{4} \hat{s}}{U T^{2}}\left[8+\frac{9 T}{U}+\frac{8 U}{T}\right]\right\}
\end{gathered}
$$

with

$$
T=\hat{t}-m_{Q}^{2}, \quad U=-\hat{s}-T
$$

Similarly for the process $\gamma+\bar{q} \rightarrow H_{Q}+X$, we have

$$
\frac{d \tilde{\sigma}_{R}^{(i)}}{d \hat{t}}(\hat{s}, \hat{t})=\frac{2 \pi \alpha \alpha_{s}^{2} e_{Q}^{2} m_{Q}^{2}}{3 \hat{s}^{2}} \mathcal{C}^{(i)} \sum_{j=1}^{5} w_{j}^{(i)} \tilde{\mathcal{B}}_{j}
$$

where 


$$
\begin{aligned}
\tilde{\mathcal{B}}_{1}= & \tilde{\mathcal{B}}_{2}=\frac{1}{4 \hat{s}}\left\{\frac{2 m_{Q}^{4} \hat{s}^{3}}{T^{3} U^{2}}\left(1+\kappa \frac{T}{\hat{s}}\right)+\frac{m_{Q}^{2} \hat{s}}{U^{2}}\left[\frac{2(1+\kappa) \hat{s}}{T}+4 \kappa+\frac{\kappa^{2} T}{\hat{s}}-\frac{\hat{s}^{3}}{T^{3}}\right]-\frac{(\hat{s}+\kappa \hat{s} T)^{2}}{\hat{s} U}\right\}, \\
\tilde{\mathcal{B}}_{3}= & \tilde{\mathcal{B}}_{4}=\frac{1}{4 \hat{s}}\left\{\frac{m_{Q}^{2} \hat{s}}{U^{2}}\left[4 \kappa(3+\kappa)+\frac{s^{3}}{T^{3}}+\frac{4(2+\kappa) s^{2}}{T^{2}}+\frac{2(3+7 \kappa) s}{T}+\frac{3 \kappa^{2} T}{s}\right]\right. \\
& \left.+\frac{6 m_{Q}^{4} \hat{s}^{2}(\hat{s}+\kappa T)}{T^{3} U^{2}}-\frac{\hat{s}}{U}\left[\left(1+\frac{\kappa T}{\hat{s}}\right)^{2}\left(1+\frac{2 U^{2}}{T^{2}}\right)\right]\right\},
\end{aligned}
$$

with $\mathcal{C}^{(1,8)}$ given in Eq.(25). In above results polarizations of initial states are averaged.

Our results contain 8 parameters in general which are defined as integrals with matrix elements of HQET. They are universal, i.e., they do not depend on a specific process. With our results one can check that the ratio is a constant:

$$
\left.\frac{d \sigma\left(g+\bar{q} \rightarrow H_{Q}+X\right)}{d \sigma\left(g+g \rightarrow H_{Q}+X\right)}\right|_{\hat{t} \rightarrow-|\hat{t}|_{\text {min }}} \sim \text { const. }
$$

i.e., the ratio does not depend on $\hat{s}$, hence the contribution from quark recombination is not suppressed by inverse of certain power of $\hat{s}$, and it can give a significant contribution. This contribution is only suppressed by the parameters given in Eq.(49).

The 8 parameters can be effectively reduced to 4 because of the property of $\mathcal{B}_{1}^{(1,8)}=\mathcal{B}_{2}^{(1,8)}$ and $\mathcal{B}_{3}^{(1,8)}=\mathcal{B}_{4}^{(1,8)}$. One can show this property without explicit calculation. With the expansion in Eq.(41) the last diagram in Fig.1. will not contribute. Taking any contribution from the first 4 diagrams in Fig.1, e.g., the interference between amplitudes from Fig.1a and Fig.1b, the coefficient of $w_{1}^{(1)}$ can be written as a trace:

$$
\mathcal{B}_{1}^{(1)} \sim \operatorname{Tr}\left\{\cdots I \gamma \cdot p_{2} I \cdots\right\}
$$

where $\cdots$ denote polynomials of products of $\gamma$ matrices, $I$ denotes $4 \times 4$ unit matrix coming from Eq.(43). Since we do not observe the polarization of initial hadrons, the spin of the initial light quark $\bar{q}\left(p_{2}\right)$ is averaged, it results in the factor $\gamma \cdot p_{2}$. The same leads to the coefficient of $w_{2}^{(1)}$ :

$$
\mathcal{B}_{2}^{(1)} \sim \operatorname{Tr}\left\{\cdots\left(-\gamma_{5}\right) \gamma \cdot p_{2} \gamma_{5} \cdots\right\}
$$

it is clear that both coefficient are same. If initial hadrons are polarized, the coefficients are in general not the same. To illustrate this, we consider the case where only the hadron containing the light quark $\bar{q}\left(p_{2}\right)$ is transversally polarized with the polarization vector $S_{T}^{\mu}$ and another initial hadron is unpolarized. In this case spin-dependent parts of perturbative coefficients, denoted as $\mathcal{B}_{s i}^{(1,8)}(i=1,2,3,4)$, can be calculated by replacing $\gamma \cdot p_{2}$ with $i \sigma_{\mu \nu} \gamma_{5} p_{2}^{\mu} S_{T}^{\nu}$, the contribution to the spin-dependent part of the cross section is a convolution with the so-called transversity distribution function instead of usual parton distribution functions [26]. With the replacement one can easily find that spin-dependent parts of perturbative coefficients have the properties $\mathcal{B}_{s 1}^{(1,8)}=-\mathcal{B}_{s 2}^{(1,8)}$ and $\mathcal{B}_{s 3}^{(1,8)}=-\mathcal{B}_{s 4}^{(1,8)}$, in contrast to the spin-independent part. Hence, if the spin-dependent parts of perturbative coefficients are not zero, we will have 8 nonperturbative parameters which lead to different contributions to the cross section. With symmetries one can also identify when these spin-dependent parts are zero in this case. With the rotational symmetry, the spin-dependent 
part of differential cross-sections consists of two parts, one is proportional to $\mathbf{S}_{\mathbf{T}} \cdot \mathbf{k}$, while another is proportional to $\mathbf{S}_{\mathbf{T}} \cdot(\mathbf{P} \times \mathbf{k})$, where $\mathbf{P}$ is the three momentum of the polarized hadron. If the parity is conserved, the term proportional to $\mathbf{S}_{\mathbf{T}} \cdot \mathbf{k}$ is zero. With the time-reversal symmetry one can show that the term proportional to $\mathbf{S}_{\mathbf{T}} \cdot(\mathbf{P} \times \mathbf{k})$ is zero if no absorptive parts in amplitudes. Further, one can show with arguments from chirality that the two terms are proportional to $m_{Q}$. Therefore, in the case considered here, we will have effectively 4 parameters only provided that there is no parity-violating interaction and absorptive part in partonic processes. Since parityviolating interactions can be involved and an absorptive part can also appear beyond tree-level, we have in general 8 parameters in differential cross-section. Similarly, one can also show that the 8 parameters lead to different contributions in the case if all initial hadrons are polarized. It should be noted that in the above discussion the polarization of the final hadron, if it is not spin-less, is summed.

Because all these parameters are unknown, a numerical prediction can not be made. As an estimation, one may use vacuum saturation for $w_{i}^{(1)}(i=1, \cdots, 4)$, i.e., the color singlet parts, to relate them to wave functions of $H_{Q}$. However, the approximation of the vacuum saturation is not well established because $\bar{q}$ is a light quark, and also the approximation definitely does not apply for the color octet part, i.e., for $w_{i}^{(8)}(i=1, \cdots, 4)$. With the definitions of these parameters one may use nonperturbative methods like QCD sum rules to study them, or they can be extracted from experiment. Once their numerical values are known, numerical predictions can be made with our results. In this work we do not make an attempt to fit experimental results with our results for determining these parameters, because in [18-20] it is shown that one can already describe the leading particle effect observed in experiment by keeping contributions with $w_{2,3}^{(1,8)}$ and neglecting other contributions. The relation between the parameters in [19] and ours can be identified:

$$
\begin{aligned}
\rho_{1}\left(Q \bar{q}\left({ }^{1} S_{0}\right) \rightarrow H_{Q}\right) & =\frac{1}{2} w_{2}^{(1)}, & \rho_{1}\left(Q \bar{q}\left({ }^{3} S_{1}\right) \rightarrow H_{Q}\right) & =\frac{1}{2} w_{3}^{(1)}, \\
\rho_{8}\left(Q \bar{q}\left({ }^{1} S_{0}\right) \rightarrow H_{Q}\right) & =\frac{3}{8} w_{2}^{(8)}, & \rho_{8}\left(Q \bar{q}\left({ }^{3} S_{1}\right) \rightarrow H_{Q}\right) & =\frac{3}{8} w_{3}^{(8)} .
\end{aligned}
$$

It should be noted that in the contributions related to $w_{2,3}^{(1,8)}$ the pair $Q \bar{q}$ is in ${ }^{1} S_{0}$ and ${ }^{3} S_{1}$ state, respectively. Our results of these contributions to the cross-section are exactly the same as those given in $[19,20]$. From our general analysis one can see that the pair $Q \bar{q}$ can form a scalar, a pseudoscalar, a vector, and a pseudovector state. All of them can lead to contributions to the leading particle effect, which can be predicted with the above results by using Eq.(28) or Eq.(29). If the light quark $\bar{q}$ is replaced with a heavy quark, one can use NRQCD factorization [21] to make prediction, then at leading order of the factorization, the $Q \bar{q}$ can only form a pseudoscalar or vector state, i.e., ${ }^{1} S_{0^{-}}$and ${ }^{3} S_{1}$ state. In this approach motivated by NRQCD factorization only terms with $w_{2,3}^{(1,8)}$ will remain, while other terms are at higher order in the approach. This approach is used in [18-20]. With this approach for polarized beams one will still have 4 parameters in contrast to our results containing 8 parameters.

\section{Summary}

In this work we have made a general analysis for the effect of quark recombination in inclu- 
sive production of a heavy meson. Through these effects the leading particle effect observed in experiment can be explained. In the contributions of quark recombination to differential cross section we factorize perturbative- and nonperturbative parts in two different cases. One is for large transverse momentum of the produced hadron, where the transverse momentum is so large that any quark mass can be neglected. In this case we find the nonperturbative effect of quark recombination is parameterized by six functions defined with four quark operators at twist- 4 . Four of these functions contribute to leading particle effect, if the initial state is unpolarized. The observed effect of leading particle is of charmed hadron, one can take the charm quark as a heavy quark and perform the factorization with HQET. In this case the nonperturbative part consists of 8 parameters, which are defined as integrals of matrix elements with 4-quark operators in HQET. These parameters represent the transition of a quark pair $Q \bar{q}$ in different states into the produced hadron, the pair can be in color singlet or color octet state and forms different states as scalar, pseudoscalar, vector and pseudovector state. The results obtained here hold not only for large transverse momentum, but also for small transverse momentum. Our results are different than those obtained with a factorization motivated by NRQCD [18-20], in which the pair only forms pseudoscalar or vector state, although for unpolarized beams both results contain the same number of nonperturbative parameters. The perturbative part in both types of factorization are calculated at tree-level for hadro- and photoproduction.

The nonperturbative functions or parameters are unknown yet. This fact prevents us from a numerical prediction for leading particle effect. But they can be studied by nonperturbative methods like QCD sum rules or models, or they can be extracted from experimental results. In this work we have not tried to extract the eight parameters from experimental results, because it is shown in [18-20] that one can already describe the leading particle effect observed in experiment

only by keeping contributions of $w_{2,3}^{(1,8)}$, and contributions of $w_{1,4}^{(1,8)}$ in our result is proportional to the contributions of $w_{2,3}^{(1,8)}$, respectively. Hence, with effects of all possible states of $Q \bar{q}$ the leading particle effect can also be generated and can be described. It should be noted that the leading particle effect of charmed baryon can be also analyzed in a similar way presented in this work, results will be published elsewhere [27].

\section{Acknowledgements}

We would like to thank Prof. E. Braaten, Prof. Y.Q. Chen, Dr. Yu Jia and Prof. J.S. Xu for discussions, Z.G.Si would like to thank members in the theoretical physics group of Shandong University for their useful discussions. The work of J.P. Ma is supported by National Nature Science Foundation of P. R. China with the grand No. 19925520. 


\section{REFERENCES}

[1] J.C. Collnis, D.E. Soper and G. Stermann, in Pertubative Quantum Chromodynamics, Ed. A.H. Mueller, World Scientific, Singapor, 1989

[2] K.P.Das and R.C. Hwa, Phys. Lett. B68 (1977) 459, ibid. B73 (1977) 504.

[3] E.L. Berger, T. Gottschalk and D.W. Siver, Phys. Rev. D23 (1981) 99

[4] E.M. Aitala et al., E791 Collaboration, Phys. Lett. B371 (1996) 157, ibid. B411 (1997) 230, ibid. Phys. Lett. B495 (2000) 42

[5] G.A. Alves et al., E769 Collaboration, Phys. Rev. Lett. 72 1994) 812, ibid. 77 (1996) 2392

[6] M. Adamovich et al., BEATRICE Collaboration, Nucl. Phys. B495 (1997) 3, M. Adamovich et al., WA82 Collaboration, Phys. Lett. B305 (1993) 402

[7] F.G. Garcia et al., SELEX Collaboration, Phys. Lett. B528 (2002) 49

[8] M. Adamovich et al., WA89 Collaboration, Eur. Phys. J. C8 (1999) 593

[9] J.C. Anjos et al., Tagged Photon Sectrometer Collaboration, Phys. Rev. Lett. 62 (1989) 513

[10] M.P. Alvarez et al., NA14/2 Collarboration, Z. Phys. C60 (1993) 53

[11] P.L. Frabetti et al., E687 Collarboration, Phys. Lett. B370 (1996) 222

[12] E. Norrbin and T. Sjöstrand, Phys. Lett. B442 (1998) 407

[13] R. Vogt and S.J. Brodsky, Nucl. Phys. B478 (1996)

[14] P. Nason, S. Dawson and R.K. Ellis, Nucl. Phys. B327 (1989) 49, ibid. B335 (1989) 260(E)

[15] W. Beenakker et al., Phys. Rev. D40 (1989) 54

[16] W. Beenakker et al., Nucl. Phys. B351 (1991) 507

[17] S. Frixione et al., Nucl. Phys. B431 (1994) 453

[18] E. Braaten, Y. Jia and T. Mehen, Phys. Rev. Lett. 89 (2002) 122002

[19] E. Braaten, Y. Jia and T. Mehen, Phys. Rev. D66 (2002) 014003

[20] E. Braaten, Y. Jia and T. Mehen, Phys. Rev. D66 (2002) 034003

[21] G.T. Bodwin, E. Braaten, and G.P. Lepage, Phys. Rev. D51, 1125 (1995); Erratum:ibid., D55, 5853 (1997)

[22] N. Isgur and M.B. Wise, Phys. Lett. B232 (1989) 113, ibid. B237 (1990) 527

E. Eichten and B. Hill, Phys. Lett. B234 (1990) 511

B. Grinstein, Nucl. Phys. B339 (1990) 253

H. Georgi, Phys. Lett. B240 (1990) 447

[23] J.P. Ma, Nucl.Phys. B506 (1997) 329

[24] J.P. Ma, Nucl.Phys. B622 (2002) 416

[25] R.K. Ellis, W. Furmanski and R. Petronzio, Nucl. Phys. B207 (1982) 1, ibid. B212 (1983) 22

R.L. Jaffe and M. Soldate, Phys. Lett. B105 (1981) 467, Phys. Rev. D26 (1982) 49

J.-W. Qiu, Phys. Rev. D42 (1990) 32

[26] R.L. Jaffe and X.D. Ji, Phys.Rev.Lett. 67 (1991) 552

R.L. Jaffe, X.D. Ji, Nucl.Phys. B375 (1992) 527

[27] C.H. Chang, J.P. Ma and Z.G. Si, work in progress 